\title{
Immunogenicity and Safety of Sinopharm COVID-19 vaccine in young mice
}

\author{
Emhemmed Ali Elgallall ${ }^{1}$, Inass A Sadawe ${ }^{2}$, Abdelmonam Hadi Elghobassa ${ }^{1}$, Abdulathim A A \\ Alshoushan', Salah M Bensaber ${ }^{3}$, Jamal Ali M Elbakay ${ }^{3}$, Nisreen H Meiqal $^{3}$, Massaud Salem \\ Maamar ${ }^{4}$, Mohammed A Gbaj ${ }^{5}$, Anton Hermann ${ }^{6}$ and Abdul M Gbaj ${ }^{3 *}$ \\ ${ }^{1}$ Food and Drug Control Centre (LFDA), Tripoli, Libya \\ ${ }^{2}$ Medical Supply Organization (MSO), Tripoli, Libya \\ ${ }^{3}$ Department of Medicinal Chemistry, Faculty of Pharmacy, University of Tripoli, Libya \\ ${ }^{4}$ Zoology Department, Faculty of Science, Tripoli University, Libya \\ ${ }^{5}$ Chemical Engineering Department, Faculty of Engineering, Tripoli University, Libya \\ ${ }^{6}$ Department of Biosciences, University of Salzburg, Salzburg, Austria \\ *Corresponding author: Abdul M Gbaj, Professor of Genetics and Biochemistry, Department of Medicinal Chemistry, Faculty of \\ Pharmacy, University of Tripoli, Libya
}

ARTICLE INFO

Received: 慧 October 18, 2021

Published: 幽 October 25, 2021

Citation: Emhemmed Ali Elgallall, Inass A Sadawe, Abdelmonam Hadi Elghobassa, Abdulathim A A Alshoushan, Abdul M Gbaj, et al., Immunogenicity and Safety of Sinopharm COVID-19 vaccine in young mice. Biomed J Sci \& Tech Res 39(4)-2021. BJSTR. MS.ID.006323.

Keywords: Sinopharm COVID-19 vaccine; mice; Coronavirus disease (COVID-19); immunogenic; safety

\section{ABSTRACT}

Background and Aim: Vaccines to prevent SARS-CoV-2 infection may be considered a promising way for reduction of the pandemic. Many different vaccines have become obtainable for use in many countries. The present study aims to evaluate the immune response and the safety of Sinopharm COVID-19 vaccine on 14 days old mice.

Materials and Methods: Our experimental study was performed on two weeks old mice, selected by random allocation. The mice were divided into three groups of 12 . Group one received a single dose of $0.5 \mathrm{ml}$ Sinopharm COVID-19 vaccine, group two received two doses of $0.5 \mathrm{ml}$ Sinopharm COVID-19 vaccine, and group three (control) received two doses of $0.5 \mathrm{ml}$ of $0.9 \% \mathrm{NaCl}$.

Results: Our study shows that Sinopharm COVID-19 vaccine is safe and induces good immunity in young mice.

Conclusions: The Sinopharm COVID-19 vaccine was safe and immunogenic in 14 days old mice. The two doses of Sinopharm COVID-19 vaccine elicit a safe antibody response in young mice. Further post-marketing toxicity studies are required to assess potential hazards for children to evaluate the histopathological characteristics.

\section{Introduction}

The severe acute respiratory syndrome caused by coronavirus 2 (SARS-CoV-2), also called COVID-19, has quickly spread over the whole world and raised severe public health distresses. The scientific society is intensely requested investigating treatments that would potentially be effective in fighting COVID-19 [1,2]. During July 2021 WHO revealed that the COVID-19 pandemic caused more than four million deaths worldwide. Vaccination has been established to limit the further spread of SARS-CoV-2 virus. Children are also vulnerable to SARS-CoV-2 infection, although they display milder clinical symptoms of the disease [3]. This susceptibility raises the possibility of transmission between family members and risk to elderly members who are more prone to the disease [4]. There are four categories of vaccines in use: whole virus, protein subunits, 
viral vectors and nucleic acid (RNA and DNA). There are additional vaccine candidates currently in the pipeline for COVID-19. All vaccines are trying to attain immunity to the virus, and some may be capable to stop transmission. By finding a proper molecule on the virus they are initiating an immune response to the antigen. In the case of COVID-19 the antigen is usually a characteristic spike protein found on the surface of the virus which assist attacks of human cells [5-7]. In case of using the entire virus (such as Sinovac and Sinopharm) it produces an immune response with the help of antigen presenting cells (APCs) such as dendritic cells (DCs) [8].

In particular, DCs have essential functions in capturing molecules, fragmenting them into smaller peptides and presenting the antigenic peptides on their major histocompatibility complex (MHC) I and II to prime T cells for the start of cellular and humoral immunity against the virus [9]. The study aims to evaluate the immune response of Sinopharm COVID-19 vaccine and its safety in young mice aged two weeks.

\section{Materials and methods}

\section{Experimental Animals}

Young Swiss Albino male mice (10 \pm 2 g) with 14 days old were used for experiments. In order to reduce the contact caused by environmental alterations and handling during behavioral studies, mice were acclimatized to the Laboratory Animal Holding Center and laboratory surroundings for three days and at least one hour before experimentation, respectively. Mice were kept under standard conditions with food (low protein diet) and water available ad libitum. The animals were housed six per cage in a light-controlled room (12 h light/dark cycle, light on 07:00 h) at $27^{\circ} \mathrm{C}$ and $65 \%$ relative humidity. All experiments were carried out between 09:30 and 15:00 h. Each test group consisted of 12 mice, and each mouse was used only once. All animal experiments were conducted according to guidelines set by the Institutional Animal Ethics Committee of the National Medical Research Centre (NMRC35/2009).

\section{Clinical and Necropsy Observations}

This study represents one constituent of the safety evaluation program for using Sinopharm COVID-19 vaccine for very young mice to assess efficacy and toxicity. The aim was to evaluate these parameters following the administration of the proposed human vaccine dose. The mice were divided into three groups of 12. Group one received a single dose of $0.5 \mathrm{ml}$ Sinopharm COVID-19 vaccine, Group two received two doses of $0.5 \mathrm{ml}$ Sinopharm COVID-19 vaccine and the second dose was given after 21 days, and group three(control) received two doses of $0.5 \mathrm{ml}$ of $0.9 \% \mathrm{NaCl}$. Mice were examined every day for 40 days. Any signs of ill health were recorded daily. Blood samples for IgM and IgG were taken from animals on day 14 and day 30 after first vaccine application. At necropsy a full macroscopic examination was performed on each animal. Organs macroscopically examined were the spleen, lungs, liver, kidney, heart, brain, testes, and ovaries.

\section{Statistical Analysis}

The difference among various treated groups and the control groups were analyzed using one-way-ANOVA followed by using unpaired Student's t-test. The results were expressed as the mean \pm SEM of the number of experiments, with $\mathrm{p}<0.05$ indicating a significant difference between groups. All $p$ values reported are for a one-tailed test. The significance level was chosen at $\alpha=0.05$.

\section{Results and Discussion}

Mice have been the most generally used animals in scientific research $[10,11]$. This could be attributed to the fact that the mouse genome is $99 \%$ identical to the human genome, and mice have similar patterns with respect to human organs and systemic physiology. The Sinopharm Beijing Covid-19 vaccine is produced by Beijing Institute of Biological Products (BIBP), subsidiary of China National Biotec Group (CNBG), they use inactive or weakened virus (19nCoV-CDC-Tan-HB02) strain as antigen which based on a form of the virus that has been inactivated or weakened so that it does not cause disease, but is still able to produce an immune response. It has been reported that the effectiveness of the vaccine is approximately $87.5 \%$ for the prevention of hospitalizations of Covid-19 patients, $65.9 \%$ for prevention of Covid-19, $90.3 \%$ for the prevention of intensive care unit admissions, and $86.3 \%$ for the prevention of Covid-19-related deaths [12]. Furthermore, children younger than 12 years old are at their crucial phase of growth and development; concern must be taken to assess the long-term effect of COVID-19 vaccine on their growth and development. In addition, children who are going to be vaccinated should have enough immunity and safety against COVID-19 vaccine [13]. It has been reported that Pfizer and Moderna messenger RNA (mRNA) vaccines studied in children older than 12 years and were found safe and effective. In addition, Pfizer and Moderna vaccines were also tested in children under 12, with the aim of involving babies from just six months old. Although teenagers only seldom get badly sick with Covid-19, they are able to spread the infection. Hence, vaccination will be able to assist stopping the pandemic [14].

Sinopharm's institute in Wuhan approved for emergency utilization on children aged between three and seventeen by the China National Biotec Group in August 2021. China began to permit people aged between three and seventeen to obtain a dose of COVID-19 vaccines in early June 2021, making it the first country to declare the endorsement of vaccines for such a young age group [15]. None of the mice used in the study showed any 
sign of abnormality or ill health throughout the 42 days postimmunization observation for the three groups after the first dose of immunization. At necropsies no macroscopic treatment related changes were observed. Antibody binding the SARS-CoV-2 spike protein was induced by vaccination, and as expected, the temporal induction of anti-spike IgM was faster than that of IgG. The mice injected with Sinopharm COVID-19 vaccine, or 0.9\% sodium chloride solutions were generally in good condition, no obvious clinical unusual symptoms were observed, and no death occurred during the observation period. The mice body weights in control and vaccine groups increased but the increase was more with the vaccinated group by around 1.5 times compared with the initial weights. There were significant differences in body weights and food intakes noted between the vaccine and negative control groups throughout the study period. Furthermore, no abnormal changes were found in the gross autopsy results of all mice investigated.

\section{Conclusion}

Our study shows that the Sinopharm COVID-19 vaccine given to 14 days old mice produces an immune response with no side effects ascertain its safety and protection efficacy against COVID-19. We highly recommend post-marketing surveillance of the vaccine safety when given for children for a longer period than that in adults.

\section{References}

1. Dhama K, Khan S, Tiwari R, Sircar S, Bhat S, et al. (2020) Coronavirus Disease 2019â€“COVID-19. Clin Microbiol Rev 33(4): e00028-20.

2. Abd El-Aziz TM, Stockand JD (2020) Recent progress and challenges in drug development against COVID-19 coronavirus (SARS-CoV-2) - an update on the status. Infect Genet Evol 83: 104327.

3. Qiu H, Wu J, Hong L, Luo Y, Song Q, et al. (2020) Clinical and epidemiological features of 36 children with coronavirus disease 2019 (COVID-19) in Zhejiang, China: an observational cohort study. Lancet Infect Dis 2020; 20(6): 689-696.

ISSN: 2574-1241

DOI: 10.26717/BJSTR.2021.39.006323

Abdul M Gbaj. Biomed J Sci \& Tech Res

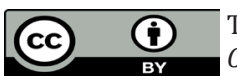

This work is licensed under Creative Commons Attribution 4.0 License

Submission Link: https://biomedres.us/submit-manuscript.php
4. Weiss P, Murdoch DR (2020) Clinical course and mortality risk of severe COVID-19. Lancet 2020; 395(10229):1014-1015.

5. Koirala A, Joo YJ, Khatami A, Chiu C, Britton PN, et al. (2020) Vaccines for COVID-19: The current state of play. Paediatr Respir Rev 2020; 35: 43-49.

6. Wang J, Peng Y, Xu H, Cui Z, Williams RO, et al. (2020) III. The COVID-19 Vaccine Race: Challenges and Opportunities in Vaccine Formulation. AAPS PharmSciTech 21(6):225.

7. Chowdhury MA, Hossain N, Kashem MA, Shahid MA, Alam A, et al. (2020) Immune response in COVID-19: A review. J Infect Public Health 13(11): 1619-1629.

8. Li W, Joshi MD, Singhania S, Ramsey KH, Murthy AK, (2014) Peptide Vaccine: Progress and Challenges. Vaccines (Basel) 2(3): 515-536.

9. Rock KL, Reits E, Neefjes J (2016) Present Yourself! By MHC Class I and MHC Class II Molecules. Trends Immunol 37(11): 724-737.

10. Justice MJ, Dhillon P (2016) Using the mouse to model human disease: increasing validity and reproducibility. Dis Model Mech 9(2): 101-103.

11. Wang S, Lai X, Deng Y, Song Y (2020) Correlation between mouse age and human age in anti-tumor research: Significance and method establishment. Life Sci 242: 117242.

12. Jara A, Undurraga EA, GonzÃilez C, Paredes F, Fontecilla T, et al. (2021) Effectiveness of an Inactivated SARS-CoV-2 Vaccine in Chile. N Engl J Med 385(10): 875-884.

13. Zou X, Cao B (2021) COVID-19 vaccines for children younger than 12 years: are we ready? Lancet Infect Dis 2021.

14. Wodi AP, Ault K, Hunter P, McNally V, Szilagyi PG, et al. (2021) Advisory Committee on Immunization Practices Recommended Immunization Schedule for Children and Adolescents Aged 18 Years or Younger United States, 2021. MMWR Morb Mortal Wkly Rep 70(6): 189-192.

15. Xia S, Zhang Y, Wang Y, Wang H, Yang Y, et al. (2021) Safety and immunogenicity of an inactivated COVID-19 vaccine, BBIBP-CorV, in people younger than 18 years: a randomised, double-blind, controlled, phase 1/2 trial. Lancet Infect Dis 2021.

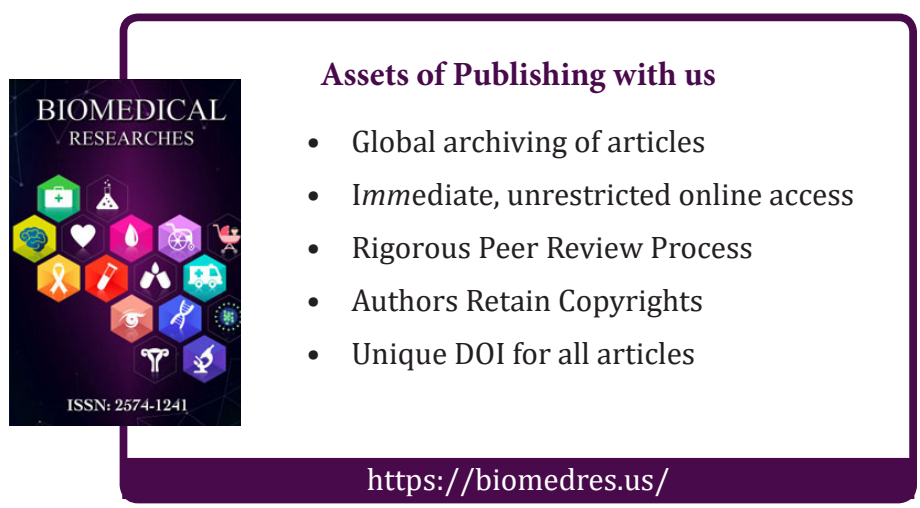

